\title{
Strategies and performances of Functional Electrical Stimulation Cycling using the BerkelBike with Spinal Cord Injury in a competition context (CYBATHLON)
}

\author{
Rik Berkelmans (1), Billy Woods (2) \\ (1) BerkelBike BV, Nieuwstraat 37, 5271 AC, Sint-Michielsgestel The Netherlands; (2) Active \\ Linx, Allia Future Business Centre, Peterborough, PE2 8AN, UK
}

This article is distributed under the terms of the Creative Commons Attribution Noncommercial License (CC BY-NC 4.0) which permits any noncommercial use, distribution, and reproduction in any medium, provided the original author(s) and source are credited.

\begin{abstract}
The functional electrical stimulation (FES) bicycle race was an event at the Cybathlon, held in Zurich October 2016. BerkelBike BV (The Netherlands) in collaboration with Imperial College London entered a spinal cord injury pilot who had tetraplegia to compete in this event. The BerkelBike Pro is a commercially available FES capable recumbent which is normally driven by the arm- and leg power. The arm cranking part was disabled. Now the tricycle must be driven using the pilots own lower limb muscles through stimulation in accordance with race rules. The bike used during the race was also adapted with a fixed gear for improved efficiency. The pilot who represented this team come second place overall in the event and attained the fastest race time of all pilots who utilised surface electrode FES. Steps can be taken to increase the race efficiency of the BerkelBikes and its FES capabilities even further.
\end{abstract}

Key Words: FES, FES ycling, Cybathlon, SCI, Tetraplegia

Eur J Transl Myol 27 (3): 255-258

The Cybathlon was an event that took place in Zurich, October 2016. This event was organised by ETH Zurich and involved six events with the aim of including individuals who were living with physical and neurological restrictions. International teams recruited 'pilots' to compete in these events, and included scientists and engineers from both academic and commercial enterprises. One of the events held at the Cybathlon was the functional electrical stimulation (FES) bike race. A pilot from the UK was entered into the FES bike race using a BerkelBike, this team was a collaboration between BerkelBike BV with support from academics at the Imperial College London (ICL).

\section{FES bike race}

This event involved two pilots riding around a $750 \mathrm{~m}$ flat track. The time taken for the pilots to reach the finish line were recorded in a first round of races which determined the pilot pairings for the finals. ${ }^{1}$ The pilots involved had spinal cord injuries (SCI) and were to move around the track using only the power from their legs, stimulated by FES. No additional pedalling assistance was allowed, this included hand cracks and pedal assist motors and drives. ${ }^{1}$ Due to no additional pedalling assistance being allowed the FES pilots needed a running start. This came in the form of a 'stumpy gate' which held the pilots and their bikes on a declining slope. Once the race started the gates opened and allowed the pilots to start rolling forward. ${ }^{1}$

\section{Pilot inclusion criteria}

The pilots for the FES bike race had to fit certain criteria $^{1}$ :

- Spinal cord injured pilots who have a lesion which has caused leg paralysis.

- ASIA A or B, with a complete loss of motor function.

- The lower motor neuron must be intact; thus, the lesion must be spastic (not atonic).

- Pilots must have sufficient voluntary control of their trunk, arms, and neck to steer the bike and stabilise the upper body while riding down a ramp and turning curves.

The BerkelBike and ICL team 3 potential pilots to choose from, all of whom were already riding a BerkelBike. A BerkelBike is an arm and leg driven tricycle. ${ }^{2}$ The candidate was selected on their ability to cycle around a test track using only power from the legs while being stimulated with FES. They suffered from a weakness in both hands, but the right hand was sufficiently weak that it had to be safely strapped to the handle bars during training and racing to ensure it stayed 
in place. The pilot chosen for the event was the candidate who could cycle the furthest using FES with legs alone. This pilot had a C5 SCI and was tetraplegic.

\section{Pilot information}

- Lesion level - C4/C5

- Age at injury - 16

- Current age - 22

- Pilot weight $-62 \mathrm{~kg}$

- Pilot height $-178 \mathrm{~cm}$

\section{Muscles stimulated during the race:}

- Quadriceps

- Hamstrings

- Gluteal muscles

\section{Stimulation Parameters}

- The stimulation program was controlled by the onboard computer.

- An encoder uses the position of the pedals to determine which muscles to stimulate

- During the race, the pilot could control the intensity of stimulations.

\section{Technology inclusion criteria}

The technology used for the race had to comply with the following criteria:

- Only passive cycling devices without actuation are allowed. The propulsion force must come from the FES activated lower limbs of the pilot.

- The FES stimulator can be commercially available or custom made. If it is custom made, it must fulfil the standard rules for electrical safety, including the latest IEC standards 606011 and 606012-10 (or similar rules applied in other countries), which describe requirements for the basic safety and essential performance of transcutaneous nerve and muscle stimulators.

- Any control strategy or stimulation pattern can be used for stimulating lower extremity muscles such as quadriceps, hamstrings, gluteal, and calf muscle groups. It is not necessary to stimulate all these muscles.

- FES stimulation intensity can be adjusted by the cycling athletes during the race so that they can apply their own strategy to minimize muscle fatigue effects.

- Surface and implanted stimulation technologies are allowed. The implantations must be medically stable for at least six months and free of complications (e.g. infections) prior to the competition

- Any number of stimulation channels are allowed.

- The FES stimulators can apply closed-loop control strategies using sensors applied to the pilots or the bike.

- The cycling device structure and functioning can be optimized for better mechanical efficiency.

- Any number and any size of wheels are allowed.
- Maximum width of the cycling device is limited to $900 \mathrm{~mm}$ to enable proper use on the ramps and in the lanes.

Cycling devices must be able to turn curves with a radius of approximately $13 \mathrm{~m}$. During the Cybathlon the pilot from BerkelBike and ICL was riding an EasyLegs Pro, ${ }^{2}$ which allows propulsion from the legs only. The frame is made from Aluminium, and the weight is $19 \mathrm{~kg}$, measuring approximately 170 by 78 by $100 \mathrm{~cm}$ in dimensions. ${ }^{2}$ The EasyLegs Pro is a recumbent tricycle with a gear hub used at the front wheel. ${ }^{2}$ A fixed gear was fitted for this event to improve pedalling efficiency. The BerkelBike Pro is a delta recumbent configuration tricycle with the pedals situated directly above the driving wheel. The seat can be changed in position relative to the longitude of the frame and in this case, was positioned slightly forward of the rear axle which has two wheels. The FES control box was situated in the centre of the handlebars of the BerkelBike and could be controlled by the pilot using buttons on this box. The FES control strategy was open-loop and the intensity of the stimulation was controlled by the pilot.

\section{FES parameters:}

- Output current range - 0 to $150 \mathrm{~mA}$.

- During the race, the pilot started at a stimulation level of $60 \mathrm{~mA}$ then worked up to the maximum of $150 \mathrm{~mA}$.

- The stimulation had a frequency of $35 \mathrm{~Hz}$.

Electrical stimulation using two electrodes per muscle was applied to the quadriceps, hamstrings and gluteal muscles of both legs of the pilot. The duration and timing of the pulses was determined by the position of the crank and the intensity was controlled by the pilot using plus and minus buttons on the stimulator attached to the steering bar in an open loop system. No further instrumentation was required during the race. The FES stimulation was applied using surface adhesive electrodes so was non-invasive. ${ }^{3-12}$

\section{Physical preparation of the pilot}

Training for the event included outdoor cycling on the BerkelBike almost every day. The pilot had been in possession of a BerkelBike for 3,5 years prior to the Cybathlon, so they were already an active user of this technology. The BerkelBike that the pilot was already in possession of was an arm and leg system, so to prepare for the Cybathlon his training included sessions in which he was restricted from using his arms. This was to let the pilot get accustomed to leg cycling only. Physiotherapist sessions were used to help manage the SCI. A track was used to periodically measure the time taken to cycle $750 \mathrm{~m}$ as a performance indicator during the training phase. The pilots diet was examined and a higher protein intake and breakfast were recommended, as these were missing previously 


\section{Performance at the Cybathlon}

The pilot obtained a silver medal during the Cybathlon, achieving the best result of all the pilots utilising adhesive surface electrodes. During the first race at the event a track time of around 4:14 minutes was obtained, whilst in training the time was regularly around 7 minutes.

\section{Discussion}

The increase in performance on the day of the event can be attributed to the conversion to a fixed gear system and updated FES protocols. During training a hub gear system was used. The limitations in cycling power output were determined by the capacity of the muscles of the pilot. A second pilot was considered for the Cybathlon who could produce much more force in his leg muscles therefore cycling faster, but he fatigued more quickly and was unable to cycle the required 750 $\mathrm{m}$ without reducing speed. The pilot was very active before his injury and thus was very motivated to become active again and find new ways of exercising, he had been using the BerkelBike for 3.5 years prior to the Cybathlon. He built up good fatigue resistance during these years and has had no injuries during training or use. As a tetraplegic, his right hand has no useful function so was strapped to the handlebars throughout the race, his left hand was used to push the buttons to control the stimulation intensity. During the final race, a bump in the track caused the pilot to be shifted in his seat, unable to shift himself back meant that performance was affected. The pilot had a faster start which dropped after the bump, the result was a time of 4:08 minutes. Improvements can be made to the seat or frame of the bike to ensure pilots are more secure in place in the future. Collaboration with other teams present at the first Cybathlon FES Cycling competition and beyond will provide further hints and tips. $^{13-20}$

\section{List of acronyms}

FES - functional electrical stimulation

SCI - spinal cord injuries

\section{Author's contributions}

$\mathrm{RB}$ and BV equally participated in experimental design, data collection, writing and revision of the manuscript.

\section{Acknowledgments}

We would like to thank Dr. Aldo Faisal and Dr Ian Radcliffe from Imperial College London for their help in organising the team and for the support during the Cybathlon. Special thanks to Paul Moore at Active Linx in also helping to prepare for the event and to Johnny Beer for participating in the race.

\section{Conflict of Interest}

The authors have no conflicts of interests.

\section{Ethical Publication Statement}

We confirm that we have read the Journal's position on issues involved in ethical publication and affirm that this report is consistent with those guidelines.

\section{Corresponding Author}

Mr. Rik Berkelmans, BerkelBike BV, Nieuwstraat 37, 5271 AC, Sint-Michielsgestel The Netherlands Email: rik@berkelbike.com

E-mails of co-author

Mr Billy James Woods, Activelinx, E-mail: billy@activelinx.co.uk

\section{References}

1. Cybathlon (2016) Races \& Rules, Championship for robot assisted athletes, ETH Zurich.

2. BerkelBike BV (2017), Sint-Michielsgestel, Netherlands.

3. Pigna E, Greco E, Morozzi G, et al. Denervation does not Induce Muscle Atrophy Through Oxidative Stress. Eur J Transl Myol 2017;27(1):6406. doi: 10.4081/ejtm.2017.6406. eCollection 2017 Feb 24.

4. Pette D, Vrbová G. The Contribution of Neuromuscular Stimulation in Elucidating Muscle Plasticity Revisited. Eur J Transl Myol. 2017 Feb 24;27(1):6368. doi: 10.4081/ejtm.2017.6368. eCollection 2017 Feb 24. Review.

5. Aksöz EA, Laubacher M, Binder-Macleod S, Hunt KJ. Effect of Stochastic Modulation of Inter-Pulse Interval During Stimulated Isokinetic Leg Extension. Eur J Transl Myol 2016;26(3):6160. eCollection 2016 Jun 13.

6. Guimarães JA, da Fonseca LO, Dos Santos-CoutoPaz CC, et al. Towards Parameters and Protocols to Recommend FES-Cycling in Cases of Paraplegia: A Preliminary Report. Eur J Transl Myol 2016;26(3):6085. eCollection 2016 Jun 13.

7. Valtin M, Kociemba K, Behling $\mathrm{C}$, et al. RehaMovePro: A Versatile Mobile Stimulation System for Transcutaneous FES Applications. Eur J Transl Myol 2016;26(3):6076. eCollection 2016 Jun 13.

8. Malešević J, Štrbac $M$, Isaković $M$, et al. Evolution of Surface Motor Activation Zones in Hemiplegic Patients During 20 Sessions of FES Therapy with Multi-pad Electrodes. Eur J Transl Myol 2016;26(2):6059. doi: 10.4081/ejtm.2016.6059. eCollection 2016 Jun 13.

9. Muthalib M, Kerr G, Nosaka K, Perrey S. Local Muscle Metabolic Demand Induced by Neuromuscular Electrical Stimulation and Voluntary Contractions at Different Force Levels: A NIRS Study. Eur J Transl Myol 2016 Jun 13;26(2):6058. doi: 10.4081/ejtm.2016.6058. eCollection 2016 Jun 13. 


\section{FES Cycling using the BerkelBike in SCI}

Eur J Transl Myol 27 (4): 255-258

10. Willand MP. Electrical Stimulation Enhances Reinnervation After Nerve Injury. Eur J Transl Myol 2015;25(4):243-8. doi: 10.4081/ejtm.2015.5243. eCollection 2015 Aug 24. Review.

11. Ravara B, Gobbo V, Carraro U, et al. Functional Electrical Stimulation as a Safe and Effective Treatment for Equine Epaxial Muscle Spasms: Clinical Evaluations and Histochemical Morphometry of Mitochondria in Muscle Biopsies. Eur J Transl Myol 2015;25(2):4910. doi: 10.4081/ejtm.2015.4910. eCollection 2015 Mar 11.

12. Lømo T, Westgaard RH, Hennig R, Gundersen K. The Response of Denervated Muscle to LongTerm Electrical Stimulation. Eur J Transl Myol 2014;24(1):3300. doi: 10.4081/ejtm.2014.3300. eCollection 2014 Mar 31.

13. Azevedo Coste C, Bergeron V, Berkelman R, et al. Comparison of strategies and performance of functional electrical stimulation cycling in spinal cord injury pilots for competition in the first ever Cybathlon. Eur J Transl Myol 2017;27:251-4.

14. Laubacher M, Aksöz EA, Bersch I, Hunt KJ. The road to Cybathlon 2016 - Functional electrical stimulation cycling Team IRPT/SPZ. Eur J Transl Myol 2017;27:259-64.
15. Sijobert B, Fattal C, Daubigney A, Azevedo-Coste B. Participation to the first Cybathlon: an overview of the FREEWHEELS team FES-cycling solution. Eur J Transl Myol 2017;27:265-71.

16. Guimarães JA, Oliveira da Fonseca L, de Sousa AC, et al. FES Bike Race preparation to Cybathlon 2016 by EMA team: a short case report. Eur J Transl Myol 2017;27:272-78.

17. Metani A, Popovi?-Maneski L, Mateo S, et al. Functional electrical stimulation cycling strategies tested during preparation for the First Cybathlon Competition - a practical report from team ENS de Lyon. Eur J Transl Myol 2017;27:279-88.

18. McDaniel J, Lombardo LM, Foglyano KM, et al. Cycle Training Using Implanted Neural Prostheses: Team Cleveland. Eur J Transl Myol 2017;27:289-94.

19. Arnin J, Yamsa-ard T, Triponyuwasin P, Wongsawat Y. Development of practical functional electrical stimulation cycling systems based on an electromyography study of the Cybathlon 2016. Eur J Transl Myol 2017;27:295301.

20. Leung WC, Tong RKY, Wang X, et al. The Effectiveness of Functional Electrical Stimulation (FES) in On-Off Mode for enhancing the cycling performance of Team Phoenix at 2016 Cybathlon. Eur J Transl Myo 2017;27:302-6. 